\title{
ANOTHER CHARACTERIZATION OF TREES
}

\author{
L. E. WARD, JR.
}

(Communicated by Dennis Burke)

\begin{abstract}
It is proved that a continuum is a tree if and only if each two connected subsets meet in a connected set.
\end{abstract}

In this note a continuum is a compact connected Hausdorff space. A tree is a continuum in which distinct points are never conjugate [6], i.e., any two distinct points are separated by the deletion of some third point. A dendrite is a metrizable tree.

The literature is replete with characterizations of dendrites, but it is frequently the case that these characterizations are invalid for trees. Recently the author [5] has observed that if $P$ is a property which characterizes dendrites among metrizable continua, then it is often the case that an arbitrary continuum is a tree if and only if it satisfies $P$ hereditarily, i.e., if every subcontinuum satisfies $P$. Therefore one can expect that a property which characterizes dendrites among metrizable continua, and which is intrinsically hereditary, will also characterize trees. To this end let us consider the "connected intersection property." A connected space satisfies the connected intersection property if the intersection of any two of its connected sets is connected. It is obvious that this property is hereditary, and it is known [6] that a metrizable continuum is a dendrite if and only if it satisfies the connected intersection property. The author knows of no published proof which is not highly metric in nature, i.e., in which the second countability of the continuum is employed in a crucial way so that there is no hope of a direct generalization to arbitrary continua. It is the purpose of this note to provide a new proof, valid in the nonmetrizable case.

It should be noted that the connected intersection property has been studied in general connected spaces by Whyburn [7] and Kok [3], especially in the locally connected case. Whyburn first proved that in a locally connected, connected Hausdorff space distinct points are never conjugate if and only if the space satisfies the connected intersection property. It is curious that the compact, nonlocally connected case has not been considered. The main difficulty

Received by the editors August 21, 1989 and, in revised form, February 22, 1990.

1980 Mathematics Subject Classification (1985 Revision). Primary 54F20, 54F50, 54F65.

Key words and phrases. Tree, tree-like, dendrite, connected intersection property, cutpoint, noncutpoint, composant, hereditarily decomposable. 
appears to hinge on the need to show that a continuum with the connected intersection property cannot contain an indecomposable continuum. This is easy to see in the metrizable case, using the standard result that an indecomposable metrizable continuum has uncountably many composants. For nonmetrizable continua this result is not available, as Bellamy has demonstrated [2]. However, Bellamy has also proved [1] that an indecomposable continuum must contain an indecomposable subcontinuum with at least $c$ composants, and the application of this theorem is an essential ingredient of the proof given here.

Lemma 1. If $M$ is a continuum with the connected intersection property, then $M$ is hereditarily decomposable.

Proof. Suppose $M$ contains a nondegenerate indecomposable continuum $K$. By a theorem of Bellamy [1, Corollary 5] $K$ contains $L$, an indecomposable continuum with at least $c$ composants. Let $C$ and $C^{\prime}$ be distinct (and hence disjoint) composants of $L$, and let $p$ and $q$ be distinct elements of $C^{\prime}$. Since $C$ is dense in $L$, the set $C \cup\{p, q\}$ is connected. Moreover, the connected sets $C \cup\{p, q\}$ and $C^{\prime}$ meet in $\{p, q\}$, contradicting the connected intersection property.

Hereafter we use the term arc in its generalized sense; that is, an arc is a continuum with exactly two noncutpoints.

Lemma 2. If $M$ is a continuum with the connected intersection property and if $M$ is irreducible about the two point set $\{a, b\}$ then $M$ is an arc with noncutpoints $a$ and $b$.

Proof. It is immediate that $M$ is hereditarily unicoherent; therefore if $x$ and $y$ are elements of $M$ then there is a unique subcontinuum $[x, y]$ of $M$ which is irreducible about $\{x, y\}$. In particular, $M=[a, b]$. To establish the lemma it is enough to show that if $p \in M-\{a, b\}$ then $[a, p] \cap[p, b]=\{p\}$.

Suppose there exists $q \in M-\{p\}$ such that $[a, q]=[a, p]$. Define

$$
S=\bigcup\{[a, z]: z \in[a, p] \text { and }[a, z] \neq[a, p]\} \text {. }
$$

Clearly, $S$ is connected and $p$ and $q$ lie in $M-S$.

We claim that $S \cup\{p, q\}$ is connected. It suffices to show that $p$ and $q$ are members of $\bar{S}$. If $p \notin \bar{S}$ then there is an open set $U$ such that $p \in U$ and $\bar{U} \cap S=\varnothing$. Let $C$ denote the component of $[a, p]-U$ which contains $a$. It follows that there exists $z \in \bar{C} \cap \partial U$; obviously $[a, z] \subset \bar{C}$ and hence $z \in S$. But $z \in \partial U \subset \bar{U}$, contrary to the fact that $\bar{U} \cap S=\varnothing$. Therefore $p \in \bar{S}$ and, by an identical argument, $q \in \bar{S}$.

We claim that $[p, q] \cap S=\varnothing$. Suppose on the contrary that there exists $z \in[p, q] \cap S$. By Lemma $1,[p, q]$ is decomposable, say $[p, q]=A \cup B$ where $A$ and $B$ are subcontinua with $p \in A-B$ and $q \in B-A$. If $z \in A$ then $[a, p] \subset[a, z] \cup[z, p] \subset S \cup A$, and hence $q \notin[a, p]$, a contradiction. If $z \in B$ then a similar contradiction ensues.

From these two claims it follows at once that the connected sets $S \cup\{p, q\}$ and $[p, q]$ meet in $\{p, q\}$, contradicting the connected intersection property. 
Consequently there exists no $q \in M-\{p\}$ with $[a, q]=[a, p]$; by an identical argument there is no $q \in M-\{p\}$ with $[q, b]=[p, b]$.

Suppose there exists $q \in[a, p] \cap[p, b]$ with $q \neq p$. Then $[a, q]$ is properly contained in $[a, p]$ and $[q, b]$ is properly contained in $[p, b]$. But then

$$
[a, q] \cup[q, b] \varsubsetneqq[a, p] \cup[p, b]=M,
$$

and this contradicts the irreducibility of $M$ about $\{a, b\}$. This completes the proof of Lemma 2.

Theorem. A continuum is a tree if and only if it satisfies the connected intersection property.

Proof. A tree is locally connected [4], and Whyburn [7] has shown that a tree satisfies the connected intersection property.

For the converse, suppose $X$ is a continuum satisfying the connected intersection property and let $x$ and $y$ be distinct elements of $X$. By Lemma 2 the irreducible subcontinuum $[x, y]$ is an arc. We will show that if $z \in$ $[x, y]-\{x, y\}$, then $z$ separates $x$ and $y$.

For each $t \in X-\{z\}$, let $A_{t}=\{a \in X: z \notin[a, t]\}$. Thus $A_{t}$ is simply the arc-component of $X-\{z\}$ which contains $t$, and $A_{t}=A_{s}$ if and only if $s \in A_{t}$. We claim that $A_{t}$ is an open set. Suppose there exists $w \in A_{t} \cap \overline{X-A_{t}}$; then there is a net $w_{\alpha}$ in $X-A_{t}$ with $w_{\alpha} \rightarrow w$. It follows that $\bigcup\left\{\left[z, w_{\alpha}\right]\right\} \cup\{w\}$ is a connected set, so by the connected intersection property we infer that $[z, w] \subset$ $\bigcup\left\{\left[z, w_{\alpha}\right]\right\} \cup\{w\}$. If $w^{\prime} \in[z, w]-\{z, w\}$ then there is an $\alpha$ such that $w^{\prime} \in\left[z, w_{\alpha}\right]-\{z\}$ and hence $w_{\alpha} \in A_{w^{\prime}}=A_{t}$. This is a contradiction and therefore $A_{t}$ is an open set.

Now $A_{x}$ and $\bigcup\left\{A_{t}: A_{t} \neq A_{x}\right\}$ are disjoint open sets containing $x$ and $y$ respectively, and their union is $X-\{z\}$. Hence $z$ separates $x$ and $y$.

\section{REFERENCES}

1. D. P. Bellamy, Composants of Hausdorff indecomposable continua: a mapping approach, Pacific J. Math. 47 (1973), 303-308.

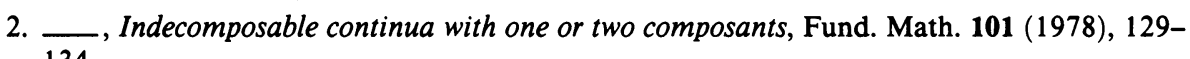
134.

3. H. Kok, Connected orderable spaces, Math. Centre Tracts, no. 49, Amsterdam, 1973.

4. L. E. Ward, Jr., A note on dendrites and trees, Proc. Amer. Math. Soc. 5 (1954), 992-994.

5. _ـ A new characterization of trees, Proc. Amer. Math. Soc. 104 (1988), 1252-1255.

6. G. T. Whyburn, Analytic topology, Colloq. Publ., vol. 28, Amer. Math. Soc., Providence, RI, 1942.

7. $\frac{}{387}$, Cutpoints in general topological spaces, Proc. Nat. Acad. Sci. U.S.A. 61 (1968), 380387.

Department of Mathematics, University of Oregon, Eugene, Oregon 97403 